Proyecciones

Vol. 26, No 3, pp. 237-244, December 2007.

Universidad Católica del Norte

Antofagasta - Chile

\title{
ON THE ALGEBRAIC DIMENSION OF BANACH SPACES OVER NON-ARCHIMEDEAN VALUED FIELDS OF ARBITRARY RANK
}

\author{
HERMINIA OCHSENIUS \\ PONTIFICIA UNIVERSIDAD CATÓLICA DE CHILE, CHILE \\ and \\ W. H. SCHIKHOF \\ RADBOUD UNIVERSITY, THE NETHERLANDS
}

Received : July 200\%. Accepted: October 2007

\begin{abstract}
Let $K$ be a complete non-archimedean valued field of any rank, and let $E$ be a $K$-Banach space with a countable topological base. We determine the algebraic dimension of $E$ (2.3, 2.4, 3.1).
\end{abstract}

AMS Classification [2000] : Primary 46510.

Keywords : Banach spaces, valued fields, algebraic dimension. 


\section{Introduction}

It is a well-known fact that the (algebraic) dimension of the Hilbert space $l^{2}$ is the power of the continuum. (See the Appendix for an elegant and general proof, kindly pointed out to us by A. van Rooij).

Now consider a Banach space $E$ with a topological base $e_{1}, e_{2}, \ldots$ over a field $K$ of any cardinality, with a non-archimedean valuation of arbitrary rank. To compute the dimension of $E$ a new approach is needed. In fact we extend Köthe's method ([2] Ch.2, Sec.9.5) used to determine the dimension of the algebraic dual of a vector space.

The results are striking. They depend strongly on whether or not $K$ is metrizable (see 2.4 and 3.1). It is also noteworthy that, if $K$ is nonmetrizable the dimension of $E$ turns out to be so small and independent of the cardinality of $K$ !

\section{Preliminaries}

We will use notations and terminology from [3], but for convenience we quote a few basics here.

Throughout $K$ is a field. Let $G$ be a totally ordered multiplicatively written abelian group with unit 1 , augmented with an element 0 satisfying $0<$ $g, 0 \cdot g=g \cdot 0=0 \cdot 0=0$ for all $g \in G$. (We point out that $G$ is not necessarily a subgroup of the positive real numbers).

A valuation on $K$ with value group $G$ is a surjective map | | : K $\rightarrow G \cup\{0\}$ satisfying

(i) $|\lambda|=0$ if and only if $\lambda=0$

(ii) $|\lambda \mu|=|\lambda||\mu|$

(iii) $|\lambda+\mu| \leq \max (|\lambda|,|\mu|)$

for all $\lambda, \mu \in K$.

The valuation is called trivial if $G=\{1\}$. The balls $B(\alpha, g):=\{\lambda \in K$ : $|\lambda-\alpha|<g\}$ where $\alpha \in K, g \in G$, induce a topology on $K$ making it into a topological field; we assume the valued field $(K,||)$ to be equipped with this topology. One introduces the notion of a Cauchy net in $K$ in a natural way. $(K,||)$ is called complete if each Cauchy net converges. We will need the following criterion on metrizability of $K$. 
Proposition 1.1. ([3] 1.4.1) $(K,||)$ is metrizable if and only if $G$ has a coinitial sequence.

A linearly ordered set $X$ without smallest element is called a $G$-module if there exists an action $(g, x) \mapsto g x$ of $G$ on $X$ that is increasing in both variables and such that $G x$ is coinitial in $X$ for all $x \in X$.

Let $E$ be a vector space over a valued field $(K,||)$, and let $X$ be a $G$-module, augmented with and element $0_{X}$ satisfying $0_{X}<x, 0 \cdot x=0 \cdot 0_{X}=0_{X}=$ $g \cdot 0_{X}$ for all $x \in X, g \in G$. For simplicity we will write 0 instead of $0_{X}$.

An $X$-norm is a map \|\|$: E \rightarrow X \cup\{0\}$ satisfying

(i) $\|x\|=0$ if and only if $x=0$

(ii) $\|\lambda x\|=|\lambda|\|x\|$

(iii) $\|x+y\| \leq \max (\|x\|,\|y\|)$

for all $x, y \in E, \lambda \in K$.

The space $(E,\|\|)$ is as usual called a normed space (more precisely, an $X$ normed space). Notice that the subset $\|E\| \backslash\{0\}:=\{\|x\|: x \in E, x \neq 0\}$ of $X$ is a $G$-module in its own right. The $X$-norm induces naturally a topology on $E$ and the notion of a Cauchy net. $(E,\|\|)$ is called a Banach space if $E$ and $K$ are complete. It is easily seen that if $(K,||)$ is metrizable then so is $(E,\|\|)$.

Let $(E,\|\|)$ be a Banach space over $K$. A system $\left\{e_{1}, e_{2}, \ldots\right\} \subset E \backslash\{0\}$ is called a topological base of $E$ if each $x \in E$ has a unique expansion as a convergent sum

$$
x=\sum_{n=1}^{\infty} \lambda_{n} e_{n} . \quad\left(\lambda_{n} \in K\right)
$$

If, in addition

$$
\|x\|=\max _{n}\left\|\lambda_{n} e_{n}\right\|
$$

it is called an orthogonal base.

Since in this paper we are concerned with the (algebraic) dimension of $E$, we recall that this is the cardinality of an algebraic base of $E$ (in the sense that each $x \in E$ can uniquely be represented as a finite linear combination of its elements). 


\section{The main result}

We prove first a well-known general fact about infinite dimensional vector spaces.

Lemma 2.1. Let $E$ be a vector space of infinite dimension $d$ over a field $K$. Let $\kappa$ be the cardinality (finite or not) of $K$, and let $\varepsilon$ be the cardinality of $E$. Then $\varepsilon=d \kappa$.

Proof. Let $\delta$ be an ordinal with cardinality $d$ and let $\left\{e_{\nu}: \nu \in \delta\right\}$ be an (algebraic) base of $E$.

For every $n \geq 1$ the cardinality of the set of elements of the form $\alpha_{1} e_{\nu_{1}}+\alpha_{2} e_{\nu_{2}}+\ldots \alpha_{n} e_{\nu_{n}}$ with $\alpha_{i} \neq 0(i=1, \ldots, n)$ is equal to $((\kappa-1) d)^{n}$. Therefore

$$
\varepsilon=\sum_{n=0}^{\infty}((\kappa-1) d)^{n}=d\left(\sum_{n=0}^{\infty}(\kappa-1)^{n}\right),
$$

since $d^{n}=d$.

If $\kappa<\aleph_{0}$ then $\sum_{n=0}^{\infty}(\kappa-1)^{n}=\aleph_{0}$ and $\varepsilon=d \aleph_{0}=d=d \kappa$.

If $\kappa \geq \aleph_{0}$ then $\sum_{n=0}^{\infty}(\kappa-1)^{n}=\kappa$ and $\varepsilon=d \kappa$.

From now on $K$ shall be an infinite field. As customary, we will often use the aleph notation for infinite cardinalities.

Lemma 2.2. Let $K$ be a valued field with value group $G \neq\{1\}$. Let $X$ be a $G$-module, let $K_{0}$ be a subfield of $K, K_{0} \neq K$. Then, for each $s, t \in X$ there exists a $\lambda \in K \backslash K_{0}$ such that $|\lambda| s<t$.

Proof. Let $G_{0}:=\left|K_{0}^{*}\right|$.

(i) Suppose $G_{0} s$ is coinitial in $X$. Then choose $\mu \in K \backslash K_{0}$. There is a $\lambda_{0} \in K_{0}^{*}$ such that $\left|\lambda_{0}\right| s<|\mu|^{-1} t$, i.e. $\left|\lambda_{0} \mu\right| s<t$. Choose $\lambda:=\lambda_{0} \mu$. Clearly, $\lambda \notin K_{0}$ (otherwise, $\mu=\lambda \lambda_{0}^{-1} \in K_{0}$, a contradiction).

(ii) Suppose $G_{0} s$ is not coinitial in $X$. Then there is a $v \in X$ such that $g_{0} s \geq v$ for all $g_{0} \in G_{0}$. In this case, choose $\lambda \in K^{*}$ such that $|\lambda| s<t$ and $|\lambda| s<v$. Then $|\lambda| \notin G_{0}$, so $\lambda \notin K_{0}$. 
Let $E$ be an infinite-dimensional Banach space with a topological base $e_{1}, e_{2}, \ldots$ over a nontrivially valued field $K$. We assume $K$ to be metrizable. We can identify $E$ with a subspace of $K^{N}$ as follows.

$$
E=\left\{\left(\xi_{1}, \xi_{2}, \ldots\right) \in K^{N}:\left|\xi_{n}\right|\left\|e_{n}\right\| \rightarrow 0\right\}
$$

(where the $\left\|e_{n}\right\|$ are in the $G$-module $\|E\| \backslash\{0\}$ ).

We want to prove the following.

Theorem 2.3. Let $E$ be an infinite-dimensional Banach space with topological base $e_{1}, e_{2}, \ldots$ over a metrizable $K$. Then the dimension of $E$ is equal to its cardinality.

Proof. Let $\aleph_{\kappa}$ be the cardinality of $K$, let $\aleph_{\varepsilon}$ be the cardinality of $E$, let $d$ be the dimension of $E$. It is enough to prove that $d \geq \aleph_{\kappa}$, since by 2.1 $\aleph_{\varepsilon}=d \aleph_{\kappa}$. Therefore we shall assume, by contradiction, that $d<\aleph_{\kappa}$. Let $\delta$ be an ordinal with cardinality $d$ and $\left\{f_{\nu}: \nu \in \delta\right\}$ an algebraic base of $E$.

For every $\nu \in \delta$ we write $f_{\nu}=\sum_{i=0}^{\infty} \alpha_{i}^{\nu} e_{i}$, and let $M:=\left\{\alpha_{i}^{\nu}: \nu \in \delta, i \in\right.$ $N\}$. Therefore the cardinality of $M$ is less than or equal to $d \aleph_{0}$. We also fix a sequence $t_{1}, t_{2}, \ldots$ in $\|E\| \backslash\{0\}$ such that $t_{n} \rightarrow 0$; we will use it to construct a chain $K_{0} \subset K_{1} \subset K_{2}, \ldots$ of subfields of $K$. In fact, let $K_{0}$ be the subfield of $K$ generated by $M$. Then, observing that the cardinality of the prime field of $K$ is at most $\aleph_{0}$, we conclude that the cardinality of $K_{0}$ is at most $d \aleph_{0}=d$. Since by assumption $d<\aleph_{\kappa}$, there exists a $\xi_{1} \in K \backslash K_{0}$, and by 2.2 we can assume that $\left|\xi_{1}\right|\left\|e_{1}\right\|<t_{1}$. We define $K_{1}:=K_{0}\left(\xi_{1}\right)$; once again $K_{1}$ has no more than $d \aleph_{0}=d$ elements, and we can pick $\xi_{2} \in K \backslash K_{1}$ such that $\left|\xi_{2}\right|\left\|e_{2}\right\|<t_{2}$. Recursively we obtain a sequence $K_{0} \subset K_{1} \subset K_{2} \subset \ldots$ of subfields, where $K_{n}=K_{n-1}\left(\xi_{n}\right)$ and $\left|\xi_{n}\right|\left\|e_{n}\right\|<t_{n}$ for all $n$.

We define the vector $\xi:=\left(\xi_{n}\right)_{n \in N}$, note that $\xi$ belongs to $E$ by construction. Write $\xi$ as a finite linear combination of the vectors in the algebraic base

$$
\xi=\sum_{j=1}^{n} \eta_{j} f_{\nu_{j}} .
$$

Let $K_{\infty}=\bigcup_{n} K_{n}$, and consider $K$ as a $K_{\infty}$ - vector space. Then $K_{\infty}$, as a subspace of $K$, has a complement $W$. Let $\varphi: K \rightarrow K_{\infty}$ be the $K_{\infty}$-linear map such that $\left.\varphi\right|_{K_{\infty}}$ is the identity map and $\left.\varphi\right|_{W}=0$. 
Then $\psi: K^{N} \rightarrow\left(K_{\infty}\right)^{N}$ defined by the formula

$\psi\left(\alpha_{1}, \alpha_{2}, \ldots\right)=\left(\varphi\left(\alpha_{1}\right), \varphi\left(\alpha_{2}\right), \ldots\right)$ is a $K_{\infty}$-linear map that is the identity on $\left(K_{\infty}\right)^{N}$. Note that, since $M \subset K_{0} \subset K_{\infty}$, the vectors $\xi$ as well as $f_{\nu}$, for any $\nu \in \delta$, belong to $\left(K_{\infty}\right)^{N}$. Now $\eta_{j} f_{\nu_{j}}=\eta_{j}\left(\alpha_{i}^{\nu_{j}}\right)_{i \in N}=\left(\eta_{j} \alpha_{i}^{\nu_{j}}\right)_{i \in N}$, and therefore $\psi\left(\eta_{j} f_{\nu_{j}}\right)=\left(\varphi\left(\eta_{j}\right) \alpha_{i}^{\nu_{j}}\right)_{i \in N}$.

Then it follows from $(*)$ that

$$
\psi(\xi)=\xi=\sum_{j=1}^{n} \eta_{j} f_{\nu_{j}}=\sum_{j=1}^{n} \varphi\left(\eta_{j}\right) f_{\nu_{j}}
$$

and, by linear independence of the set of base vectors $f_{\nu}$, we obtain that $\eta_{j}=\varphi\left(\eta_{j}\right)$, hence $\eta_{j} \in K_{\infty}$. But then, there exists an $m \in N$ such that all of the $\eta_{1}, \eta_{2}, \ldots, \eta_{n}$ belong to $K_{m}$. Then all coordinates of $\sum_{j=1}^{n} \eta_{j} f_{\nu_{j}}$ lie in $K_{m}$. Therefore for all $i \in N$ we have that $\xi_{i} \in K_{m}$, and this contradiction shows that $d \geq \aleph_{\kappa}$, which finishes the proof.

We can even say more.

Theorem 2.4. Let $E$ be an infinite-dimensional Banach space with topological base $e_{1}, e_{2}, \ldots$ over a metrizable $K$. Let $\aleph_{\kappa}$ be the cardinality of $K$, let $d$ be the dimension of $E$. Then $d=\aleph_{\kappa}^{\aleph_{0}}$.

Proof. By 1.1 there exist $\alpha_{1}, \alpha_{2}, \ldots \in K^{*}$ such that $\left|\alpha_{n}\right| \rightarrow 0$. Choose $s \in\|E\| \backslash\{0\}$ and put $t_{n}:=\left|\alpha_{n}\right| s$. Then $t_{n} \rightarrow 0$, and $E$ contains the set

$$
B=\left\{\left(\xi_{n}\right)_{n \in N}: \xi_{n} \in B_{n}\right\}
$$

where $B_{n}:=\left\{\mu \in K:|\mu|\left\|e_{n}\right\| \leq t_{n}\right\}$.

All $B_{n}$ are balls in $K$ about 0 . Now we claim that $\aleph_{\beta}$, the cardinality of $B_{n}$, is equal to $\aleph_{\kappa}$. In fact, choose $1<\left|\lambda_{1}\right|<\left|\lambda_{2}\right|<\ldots,\left|\lambda_{j}\right| \rightarrow \infty$. Then for each element $\alpha \in K$ we can choose $m \in N$ such that $\mu^{\prime}=\lambda_{m}^{-1} \alpha$ belongs to $B_{n}$. Therefore $\alpha=\lambda_{m} \mu^{\prime}$, which implies that $\aleph_{\kappa} \leq \aleph_{0} \cdot \aleph_{\beta}=\aleph_{\beta}$. The other inequality being trivial, we obtain $\aleph_{\beta}=\aleph_{\kappa}$. In particular all the balls $B_{n}$ have the same cardinality. Since $E \supset B$ and the cardinality of $B$ is $\aleph_{\kappa}^{\aleph_{0}}$, we have $\aleph_{\varepsilon} \geq \aleph_{\kappa}^{\aleph_{0}}$. As $E \subset K^{N}$, the opposite inequality is trivial.

Remark 2.5. From set theory (see [1]) we know, assuming the Axiom of Choice and the Generalized Continuum Hypothesis, that if $\kappa \neq 0$ is a limit ordinal with cofinality $\aleph_{0}$ then $\aleph_{\kappa}^{\aleph_{0}}=\aleph_{\kappa+1}=2^{\aleph_{\kappa}}$ and that in all other cases $\aleph_{\kappa}^{\aleph_{0}}=\aleph_{\kappa}$. 


\section{The case for non-metrizable $K$}

Theorem 3.1. Let $E$ be an infinite-dimensional Banach space with topological base $e_{1}, e_{2}, \ldots$ over a non-metrizable $K$. Then the dimension of $E$ is $\aleph_{0}$ and the cardinalities of $E$ and $K$ are equal.

Proof. Let $x \in E$ have the expansion $\sum_{n=0}^{\infty} \lambda_{n} e_{n}$; we will show that this is in fact a finite sum. In fact, assume $\left\|\lambda_{n_{1}} e_{n_{1}}\right\|>\left\|\lambda_{n_{2}} e_{n_{2}}\right\|>\ldots$, $\left\|\lambda_{n_{i}} e_{n_{i}}\right\| \rightarrow 0$. Since $\|E\| \backslash\{0\}$ is a $G$-module, there are $\mu_{1}, \mu_{2}, \ldots \in K^{*}$ such that $\left|\mu_{k}\right|\left\|\lambda_{n_{1}} e_{n_{1}}\right\|<\left\|\lambda_{n_{k}} e_{n_{k}}\right\|$ for all $k$. It follows that $\mu_{k} \lambda_{n_{1}} e_{n_{1}} \rightarrow 0$, hence $\left|\mu_{k}\right| \rightarrow 0$, so that $G$ has a coinitial sequence, conflicting 1.1.

We see that $E$ is the space of all finite linear combinations of $e_{1}, e_{2}, \ldots$, which is algebraically isomorphic to $\bigoplus_{N} K$ and the conclusion follows.

Remark. At first sight it may seem strange that a Banach space can have countable dimension! But one has to keep in mind that, due to nonmetrizability, the Baire Category Theorem does not apply to $E$.

\section{Appendix}

As promised in the Introduction we compute the dimension of $l^{2}$. In fact we prove more.

Proposition 4.1. Let $E$ be a Banach space over $\mathbf{R}$ or $\mathbf{C}$ with a topological base $e_{1}, e_{2}, \ldots$. Then the dimension of $E$ is the power of the continuum.

Proof. Let $L$ be either $\mathbf{R}$ or $\mathbf{C}$, with cardinality $c$. For a set $I$, let $l^{\infty}(I)$ be the $L$-vector space of all bounded functions $I \rightarrow L$. By 2.1 we only have to prove that $\operatorname{dim} E \geq c$. To this end we may assume by scalar multiplication, that $\sum_{n=1}^{\infty}\left\|e_{n}\right\|<\infty$. Then the formula

$$
\left(\xi_{1}, \xi_{2}, \ldots\right) \mapsto \sum_{n=1}^{\infty} \xi_{n} e_{n}
$$

defines a linear injection $l^{\infty}(\mathbf{N}) \rightarrow E$. Since $\mathbf{Q}$ is countable, the spaces $l^{\infty}(\mathbf{N})$ and $l^{\infty}(\mathbf{Q})$ are isomorphic. For each $t \in \mathbf{R}$, let $f_{t} \in l^{\infty}(\mathbf{Q})$ be defined by

$$
f_{t}(q)=\left\{\begin{array}{lll}
1 & \text { if } \quad q \in Q, \quad q \geq t \\
0 & \text { if } \quad q \in Q, \quad q<t
\end{array}\right.
$$


It is easily seen that the $f_{t}(t \in \mathbf{R})$ are linearly independent. Then dim $E \geq \operatorname{dim} l^{\infty}(\mathbf{N})=\operatorname{dim} l^{\infty}(\mathbf{Q}) \geq c$ and we are done.

Remark. It is not hard to see that the techniques used in Section 2, appropriately modified, may furnish another proof of the Proposition above.

\section{References}

[1] T. Jech. Set Theory. San Diego: Academic Press. U. S. A., (1978).

[2] G. Köthe. Topological Vector spaces. New York: Springer-Verlag, (1969).

[3] H. Ochsenius and W. Schikhof. Banach spaces over fields with an infinite rank valuation. In: $p$-adic Functional Analysis, Lecture Notes in pure and applied mathematics 207, edited by J. Kakol, N. De GrandeDe Kimpe and C. Perez-Garcia. Marcel Dekker, pp. 233-293, (1999).

\section{H. OCHSENIUS}

Facultad de Matemáticas

Pontificia Universidad Católica de Chile

Casilla 306 - Correo 22

Santiago

Chile

e-mail : hochsen@mat.puc.cl

and

\section{W. H. SCHIKHOF}

Department of Mathematics

Radboud University, Toernooiveld 6525 ED Nijmegen

The Netherlands

e-mail : w_schikhof@hetnet.nl 